\title{
Phase-charge duality in Josephson junction circuits: Role of inertia and effect of microwave irradiation
}

\author{
W. Guichard ${ }^{1}$ and F. W. J. Hekking ${ }^{2}$ \\ ${ }^{1}$ Institut Néel, C.N.R.S. and Université Joseph Fourier, BP 166, 38042 Grenoble, France \\ ${ }^{2}$ Université Joseph Fourier, Laboratoire de Physique et Modélisation \\ des Milieux Condensés, C.N.R.S., BP 166, 38042 Grenoble, France
}

(Dated: May 21, 2018)

\begin{abstract}
We investigate the physics of coherent quantum phase slips in two distinct circuits containing small Josephson junctions: (i) a single junction embedded in an inductive environment and (ii) a long chain of junctions. Starting from the standard Josephson Hamiltonian, the single junction circuit can be analyzed using quasi-classical methods; we formulate the conditions under which the resulting quasi-charge dynamics is exactly dual to the usual phase dynamics associated with Josephson tunneling. For the chain we use the fact that its collective behavior can be characterized by one variable: the number $m$ of quantum phase slips present on it. We conclude that the dynamics of the conjugate quasi-charge is again exactly dual to the standard phase dynamics of a single Josephson junction. In both cases we elucidate the role of the inductance, essential to obtain exact duality. These conclusions have profound consequences for the behavior of single junctions and chains under microwave irradiation. Since both systems are governed by a model exactly dual to the standard resistively and capacitively shunted junction model, we expect the appearance of current-Shapiro steps. We numerically calculate the corresponding current-voltage characteristics in a wide range of parameters. Our results are of interest in view of a metrological current standard.

PACS numbers: 74.50.+r, 74.81.Fa, 72.30.+q
\end{abstract}

\section{INTRODUCTION}

Two physical systems that can be mapped onto each other by interchanging the role of position and its canonically conjugate momentum are said to be related by duality. If the physical properties of one of the systems are known, those of its dual counterpart can be predicted by applying the set of duality transformations that accompany the position and momentum interchange and relate the parameters of the two systems. In some special cases, duality maps the system onto itself; one then speaks of self-duality. An example of a system that exhibits exact self-duality is the harmonic oscillator. More frequently one encounters systems that exhibit an approximate selfduality relating the system's asymptotic behavior in two different limiting parameter regimes.

Duality transformations have been proven useful in a variety of situations from a broad range of fields 1 including statistical mechanics, condensed matter physics and gauge-field theories. Here we will focus on the case of Josephson junctions embedded in an electromagnetic environment, where the duality associated with the conjugate charge and phase degrees of freedom has been employed to study the circuit's dissipative dynamics $2 / 3 / 4 / 5$. A Josephson junction, as depicted schematically for a current-biased situation in Fig. 1 1 a, is characterized by two competing energy scales: the Josephson coupling energy $E_{J}=\hbar I_{c} / 2 e$, proportional to the Josephson critical current $I_{c}$, and the charging energy $E_{C}=e^{2} / 2 C$, inversely proportional to the junction's capacitance $C$. A self-duality property can be used to relate the junction's behavior in the presence of a resistor $R$ in the two limiting cases $E_{J} / E_{C} \gg 1$ and $E_{J} / E_{C} \ll 1^{2|3| 4 \mid 5}$. This self- duality is only approximate, though; as we will discuss in more detail below, an exact duality transformation exists between the circuit of Fig. 17a and a different superconducting circuit containing a large junction together with an additional element: an inductance $L^{[36}$, see Fig. 1 b. This is to be expected somehow: it is customary to describe the dynamics of the current-biased circuit depicted in Fig. 1a in terms of a fictitious phase-particle of mass $C$. The dual situation Fig. 1b would then correspond to a voltage-biased circuit, the dynamics of which is that of a charge-particle of mass $L$.

Let us push the duality analysis a little further. The phase-particle for the current-biased circuit Fig. 17 moves in a potential $U(\phi)$ which is the sum of a periodic part and a linear tilt, see Fig. 2 for an example. The periodic potential induces the tunneling of Cooper pairs of charge $2 e$, its amplitude $E_{J}=\hbar I_{c} / 2 e$ sets the maximum supercurrent $I_{c}$ that can be carried by the junction. The tilt of the potential is proportional to the bias current $I_{b}$ through the junction. By duality, the charge-particle for the voltage-biased circuit Fig. 10 moves in a potential $U(q)$ which also has a periodic part and a linear tilt, see Fig. 2. Here the periodic part induces a tunneling of phase or a "phase slip" whereby the phase difference across the junction winds by an amount $2 \pi$; accordingly, one can speak of a phase-slip junction. A winding of the phase with time gives rise to a voltage over the element, and the amplitude of the periodic part of the potential sets the maximum voltage $V_{c}$ the phase-slip element can sustain. The linear tilt is proportional to the bias voltage $V_{b}$ across the junction.

Duality thus implies that the $I-V$ characteristics of the voltage-biased circuit in Fig. 1p can be obtained from 


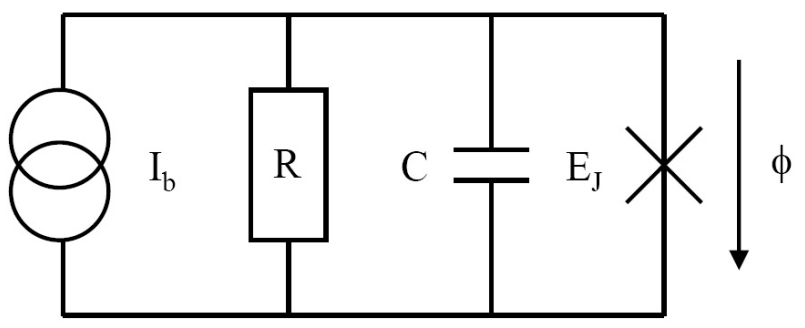

(a)

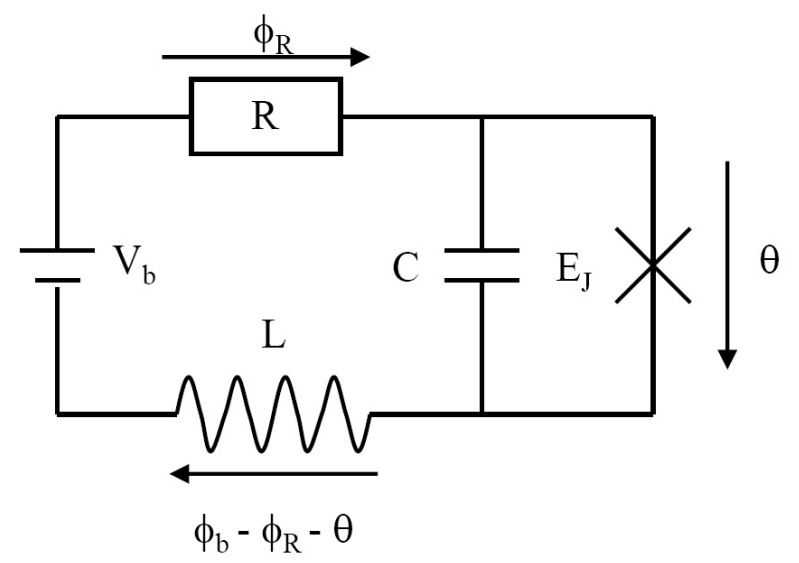

(b)

FIG. 1: (a) RCSJ-model: Current-biased Josephson junction (cross), bias current $I_{b}$, with Josephson energy $E_{J}$ shunted by a capacitor $C$ and a resistor $R$. (b) CJRL-model: Voltagebiased Josephson junction (cross), bias voltage $V_{b}$, shunted by a capacitor $C$ and in series with an inductor $L$ and a resistor $R$.

those of the current-biased circuit of Fig. 17, by simply interchanging the role of current and voltage. This has been verified experimentally in Ref!7], where the measured $I-V$ characteristics of an underdamped Josephson junction were found to be exactly dual to those of the overdamped Josephson junction measured in Ref. ${ }^{[}$, in accordance with the approximate self-duality exhibited by a Josephson junction in these respective limits.

Probably one of the most important consequences of duality in this connection is the case of a junction that is irradiated by microwaves (MW) of frequency $f$. If the MW frequency $f$ is commensurate with the frequency of the motion of the phase-particle in the periodic potential, phase-locking occurs yielding Shapiro steps 9 , in the $I-V$ characteristics at well-defined voltages that are proportional to multiples $n$ of the applied frequency $f: V_{n}=n h f / 2 e$, where $h$ is Planck's constant and $e$ the elementary charge. As frequency can be controlled with an extreme accuracy, this effect is currently used in metrology to define the voltage standard, for a review see $e^{1011}$. Observation of the dual phenomenon - phase locking for the charge-particle yielding Shapiro steps at well-defined currents that are multiples of the applied

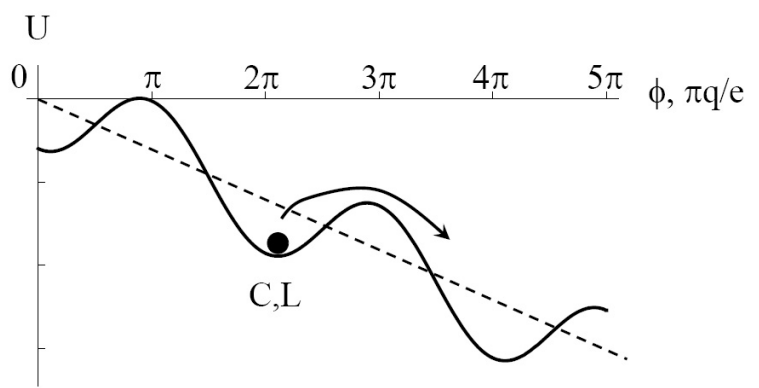

FIG. 2: Example of the tilted washboard potential $U$ as a function of phase $\phi$ and charge $\pi q / e$ for a phase-particle of mass $C$ and a charge-particle of mass $L$, respectively. The dashed line denotes the average tilt, proportional to the bias current (phase-particle) through or the bias voltage (chargeparticle) over the junction.

frequency $I_{n}=n 2 e f$ - would have far-reaching consequences for metrology, as this would enable one to define a current standard with an unprecedented precision.

Clearly, the voltage-biased circuit illustrated in Fig. $1 \mathrm{p}$ is not the only one dual to that of Fig. 1 1 . In fact, any circuit element that features appropriate phase tunneling is a possible candidate for duality. In view of experimental implementations and applications it is interesting to compare several possibilities. In a recent paper, Mooij and Nazarov $\sqrt{12}$ proposed exact duality between Fig. 1 a and a voltage-biased circuit containing a narrow superconducting wire ${ }^{13 / 14}$. As we will detail below another possibility would be to use a one-dimensional chain of Josephson junctions.

The paper is organized as follows. For pedagogical reasons and also for the sake of completeness, we start by reviewing the physics of the circuits of Fig. 1 in Section II. This enables us to demonstrate the duality principle as well as its consequences for superconducting circuits with the aid of a relatively simple example. In Section III we demonstrate that a voltage-biased chain of junctions is exactly dual to Fig. 17. The current-voltage characteristics of phase-slip junctions both in the absence and in the presence of MW irradiation are discussed in Section IV. some perspectives and experimental consequences of our theoretical study are discussed in Section $\mathrm{V}$.

\section{CHARGE-PHASE DUALITY IN CIRCUITS CONTAINING A SINGLE JUNCTION}

In this Section we wish to demonstrate that the two superconducting circuits depicted in Fig. 11are dual to each other. Specifically, we will establish the conditions under which this duality holds, treating both circuits entirely quantum mechanically. We will discuss the requirements to be met such that the classical limit can be taken, leading to the usual resistively and capacitively shunted junc- 
tion (RCSJ) mode ${ }^{15}$ for Fig. 17 and its dual counterpart, the capacitively shunted junction in series with a resistor and an inductance (CJRL) model for Fig. 1p.

\section{A. Capacitively shunted junction: phase-inertia}

We start our analysis by considering the circuit shown in Fig. 17a. It contains a current-biased Josephson junction (bias current $I_{b}$ ), shunted by a capacitance $C$ and a resistance $R$. Let $\hat{\phi}$ be the operator corresponding to the phase difference across the junction and $\hat{Q}$ the canonically conjugate charge, such that the commutator $[\hat{Q}, \hat{\phi}]=-2 i e$. The resistor induces dissipation that we will account for within the framework of the Caldeira-Leggett mode ${ }^{3 / 4 / 16117}$. Hence the circuit presented in Fig. 1a can be described by the Hamiltonian $\hat{H}=\hat{H}_{0}+\hat{H}_{B}$ where

$$
\begin{array}{r}
\hat{H}_{0}=\frac{\hat{Q}^{2}}{2 C}+U(\hat{\phi}), U(\hat{\phi})=-E_{J} \cos \hat{\phi}-\hbar I_{b} \hat{\phi} / 2 e \\
\hat{H}_{B}=\sum_{i=1}^{\infty} \frac{\hat{P}_{i}^{2}}{2}+\frac{\omega_{i}^{2}}{2}\left(\hat{X}_{i}-\frac{c_{i}}{\omega_{i}^{2}} \hat{\phi}\right)^{2} .
\end{array}
$$

Here $\hat{H}_{B}$ is the Caldeira-Leggett Hamiltonian describing a bath of oscillators with frequencies $\omega_{i}$, conjugate momenta and positions $\hat{P}_{i}$ and $\hat{X}_{i}$, the latter coupling linearly to the junction's phase operator $\hat{\phi}$ with coupling constants $c_{i}$.

From the Hamiltonian $\hat{H}$, one can obtain the equation of motion for the operator $\hat{\phi}$,

$$
\hbar C \ddot{\hat{\phi}} / 2 e+I_{c} \sin \phi=I_{b}+\delta \hat{I},
$$

where the current $\delta \hat{I}$ is related to the momenta of the oscillator bath,

$$
\delta \hat{I}=-\frac{2 e}{\hbar} \sum_{i=1}^{\infty} \frac{c_{i}}{\omega_{i}^{2}} \dot{\hat{P}}_{i}
$$

The bath momenta satisfy the equation of motion

$$
\ddot{\hat{P}}_{i}+\omega_{i}^{2} \hat{P}_{i}=c_{i} \dot{\hat{\phi}}
$$

Direct integration of Eq. (5) and substitution of the result into Eq. (4) yields

$$
\delta \hat{I}=\hat{i}(t)-\int_{0}^{t} Y\left(t-t^{\prime}\right) \hbar \dot{\hat{\phi}}\left(t^{\prime}\right) / 2 e
$$

where the first term $\hat{i}(t)$ is related to the homogeneous solution of Eq. (5); it is random in nature due to the uncertainty with respect to the bath's initial conditions. The second term is related to the particular solution of Eq. (5); it describes the response of the bath to the voltage operator $\hbar \dot{\hat{\phi}} / 2 e$ through the retarded admittance $Y(t)$ with Fourier transform

$$
Y(\omega)=\left(\frac{2 e}{\hbar}\right)^{2} \sum_{i=1}^{\infty} \frac{c_{i}^{2}}{\omega_{i}^{2}} \frac{i \omega}{(\omega+i \eta)^{2}-\omega_{i}^{2}} .
$$

Provided we choose the bath parameters $c_{i}$ and $\omega_{i}$ such that

$$
\Re \mathrm{e}[Y(\omega)]=\left(\frac{2 e}{\hbar}\right)^{2} \pi \sum_{i=1}^{\infty} \frac{c_{i}^{2}}{\omega_{i}} \delta\left(\omega^{2}-\omega_{i}^{2}\right)=1 / R,
$$

the bath's response is ohmic corresponding to that of a resistance $R$. As a result we can present Eq. (3) in the form

$$
\hbar C \ddot{\hat{\phi}} / 2 e+\hbar \dot{\hat{\phi}} / 2 e R+I_{c} \sin \hat{\phi}=I_{b}+\hat{i} .
$$

For later use, it is convenient to write Eq. (9) in a dimensionless form, dividing both sides by $I_{c}$; one then obtains

$$
d^{2} \hat{\phi} / d \tau^{2}+\sigma d \hat{\phi} / d \tau+\sin \hat{\phi}=\bar{I}_{b}+\hat{\bar{i}}
$$

where $\tau=\omega_{p} t$ with $\omega_{p}=\left(8 E_{J} E_{C}\right)^{1 / 2} / \hbar$ the junction's plasma frequency, $\sigma=\left(\hbar / 2 e I_{c} C\right)^{1 / 2} / R$ the dimensionless inverse resistance, $\bar{I}_{b}=I_{b} / I_{c}$ and $\hat{\bar{i}}=\hat{i} / I_{c}$.

Equation (9) is a non-linear quantum Langevin equation, owing its stochastic nature to the presence of the random operator $\hat{i}$. The statistics of $\hat{i}$ will be fixed by assuming the initial state of the bath to be the canonical equilibrium one at temperature $T$. This, together with the harmonic nature of the bath, implies that the statistics of the random operator $\hat{i}$ is Gaussian with average value $\langle\hat{i}\rangle=0$. In accordance with the fluctuation-dissipation theorem, the symmetrized second moment $\langle\{\hat{i}(t), \hat{i}(0)\}\rangle / 2$, where $\{\ldots, \ldots\}$ denotes the anti-commutator, is then characterized by the spectral function

$$
\begin{aligned}
S_{i}(\omega) & \equiv \int d t e^{i \omega t}\langle\{\hat{i}(t), \hat{i}(0)\}\rangle / 2 \\
& =\hbar \omega \Re \mathrm{e}[Y(\omega)] \operatorname{coth}\left(\hbar \omega / 2 k_{B} T\right) \\
& =\frac{\hbar \omega}{R} \operatorname{coth}\left(\hbar \omega / 2 k_{B} T\right) .
\end{aligned}
$$

A general analysis of the quantum Langevin equation 9 is beyond the scope of this article. Here we are interested in the classical limit of the operator equation (9) where it reduces to the well-known resistively and capacitively shunted junction (RCSJ) model, describing the classical dynamics of a fictitious phase-particle. The capacitor provides the particle's inertia; the corresponding acceleration is the capacitor's displacement current. The resistor provides both velocity-proportional damping $\sim \dot{\phi} / R$ and classical noise $i(t)$. The noise $i$ adds to the applied bias current $I_{b}$ which, together with the junction's supercurrent $I_{c} \sin \phi$ yields the external force acting on the particle. 
A classical interpretation of the operator equation (9) makes sense if we can accurately replace the operators by their respective expectation values, $\hat{\phi} \rightarrow \phi, \hat{i} \rightarrow i$. In particular, we must be allowed to replace $\langle\sin \hat{\phi}\rangle$ by $\sin \langle\hat{\phi}\rangle=\sin \phi$. For this to be correct, the uncertainty $\delta \phi$ in the phase must be small compared to the period of the sine function. The classical version of $(9)$ then describes the motion of a narrow wave packet of width $\delta \phi \ll 1$. Such a wave packet can be constructed as a superposition of extended phase states, implying an uncertainty on the level of the junction charge $\delta Q$ that exceeds the elementary charge $e$. This means in particular that the quasi-classical phase description does not capture effects associated with Coulomb blockade ${ }^{17}$.

It is useful to distinguish two cases, according the value of the parameter $\sigma$ in Eq. 100: overdamped phase dynamics, corresponding to $\sigma>1$ and underdamped phase dynamics, $\sigma<1$.

In the overdamped case, the phase dynamics is always classical as damping times are naturally short, of the order of $R C$. This is generally achieved in a low-resistance environment that avoids charge localization. Classical phase dynamics also requires that the environmental noise operator $\hat{i}$ can be treated classically 19 . This corresponds to relatively high temperatures, such that $S_{i}(\omega)=2 k_{B} T / R$, see Eq. (11). Then we can replace $\hat{i}$ by a c-number $i$ such that $\langle i\rangle=0$ and $\langle i(t) i(0)\rangle=2\left(k_{B} T / R\right) \delta(t)$; the noise is $\delta$-correlated. For this to be correct the temperature $T$ should be large compared to the characteristic frequency $1 / R C$. The overdamped limit can be analyzed, e.g., by studying the Fokker-Planck equation ${ }^{18}$ corresponding to the classical Langevin equation, as it was done for the overdamped case in Ref.19].

In the underdamped case, damping times are long and wave packet spreading becomes important. This issue is particularly relevant when the phase-particle is in the running state corresponding to a finite voltage over the junction. In the absence of damping, the uncertainty $\delta \phi$ can be kept within limits by applying classical timedependent external forces. The spreading of wave packets is governed by a rate proportional to the kinetic energy. For the fictitious phase particle this corresponds to the charging energy $E_{C}$, which is in fact the energy scale associated with charge localization leading to Coulomb blockade. We require the time-dependence of the external force to be fast on the scale $\hbar / E_{C}$. Physically this means that we work under conditions where charging effects can be ignored. For example, in the case of externally applied microwaves (see Section IV) this implies their frequency to be larger than $E_{C} / \hbar$.

\section{B. Junction in series with an inductance: charge-inertia}

Next consider the circuit depicted in Fig. 1p, where a single Josephson junction is embedded in a combined

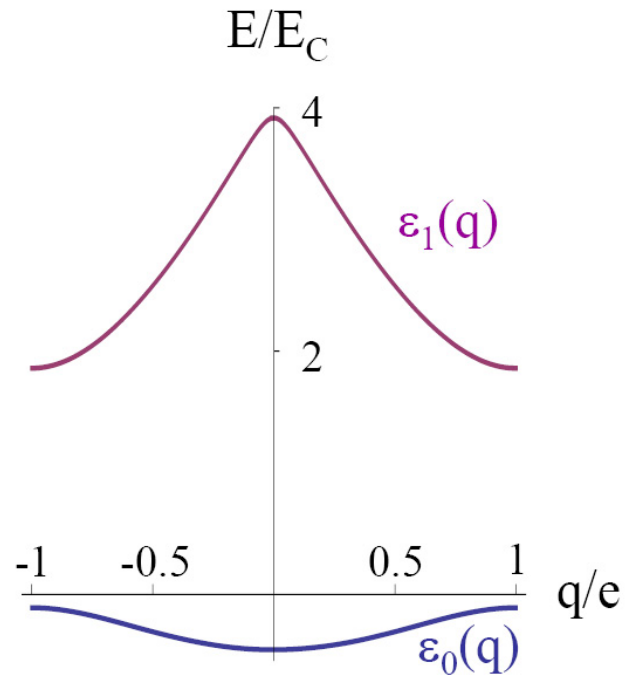

FIG. 3: Lowest two Bloch bands of the spectrum of Hamiltonian $\hat{H}_{J}$, taking the ratio $E_{J} / E_{C}=2$. Note that the bands are separated by an energy of the order of the plasma frequency, $\hbar \omega_{p}=4 E_{C}$.

inductive and resistive series environment (inductance $L$ and resistance $R)^{316}$. Its Hamiltonian is given by $\hat{H}^{\prime}=$ $\hat{H}_{0}^{\prime}+\hat{H}_{B}^{\prime}$. Here $\hat{H}_{0}^{\prime}=\hat{H}_{J}+\hat{H}_{L}$ with

$$
\hat{H}_{J}=\frac{\hat{Q}^{2}}{2 C}-E_{J} \cos \hat{\theta} ; \hat{H}_{L}=\left(\frac{\hbar}{2 e}\right)^{2} \frac{\left(\phi_{b}-\hat{\phi}_{R}-\hat{\theta}\right)^{2}}{2 L},
$$

where $\hat{\theta}$ is the phase difference across the junction, conjugate to the charge $\hat{Q}$, such that $[\hat{Q}, \hat{\theta}]=-2 i e$. The combination $\phi_{b}-\hat{\phi}_{R}-\hat{\theta}$ is the phase difference across the inductance. It contains the total phase difference across the circuit $\phi_{b}$, which is an external parameter determined by the applied voltage bias $V_{b}$, such that $\dot{\phi}_{b}=2 e V_{b} / \hbar$. The operator $\hat{\phi}_{R}$ is the phase difference across the resistor. We express it in terms of the oscillator bath positions $\hat{\Xi}_{i}$ according to the relation

$$
\hat{\phi}_{R}=\sum_{i} \lambda_{i} \hat{\Xi}_{i}
$$

with coupling constants $\lambda_{i}$. The dynamics of the bath degrees of freedom, accounting for dissipation due to the resistor, is governed by the Hamiltonian $\hat{H}_{B}^{\prime}$

$$
\hat{H}_{B}^{\prime}=\sum_{i=1}^{\infty} \frac{\hat{\Pi}_{i}^{2}}{2}+\frac{1}{2} \tilde{\omega}_{i}^{2} \hat{\Xi}_{i}^{2},
$$

where $\hat{\Pi}_{i}$ are the bath momenta conjugate to $\hat{\Xi}_{i} ; \tilde{\omega}_{i}$ are the bath frequencies.

Let us first consider the junction Hamiltonian $\hat{H}_{J}$, which describes a particle of mass $C$ moving in a periodic potential. The spectrum of this Hamiltonian consists of 
Bloch bands 20121 , see Fig. 3. We can use the so-called quasi-charge representation ${ }^{2122}$ and write $\hat{H}_{J}=\epsilon_{0}(\hat{q})$, assuming the junction's dynamics to be restricted to the lowest Bloch band $\epsilon_{0}(q)$. The commutation relation of the quasi-charge operator $\hat{q}$ and the phase $\hat{\theta}$ reads $[\hat{q}, \hat{\theta}]=-2 i e$. The physical meaning of the quasi-charge becomes clear if we consider its dynamics, which is governed by the equation of motion

$$
\dot{\hat{q}}=\frac{\hbar}{2 e L}\left(\phi_{b}-\hat{\phi}_{R}-\hat{\theta}\right)=\frac{2 e}{\hbar} \frac{d \hat{H}^{\prime}}{d \phi_{b}} .
$$

Since $\phi_{b}$ is the externally fixed phase drop over the entire circuit, the last term on the right-hand-side corresponds by definition to the total current flowing through the circuit. We next calculate $\dot{\hat{\theta}}=i\left[\hat{H}^{\prime}, \hat{\theta}\right] / \hbar$ and find

$$
\dot{\hat{\theta}}=\frac{2 e}{\hbar} \frac{\partial \epsilon_{0}(q)}{\partial q},
$$

provided that interband transitions can be ignored ${ }^{22}$. Then the operator $\partial \epsilon_{0}(q) / \partial q$ corresponds to the voltage drop over the junction. A straightforward calculation of $\ddot{\hat{q}}$ now yields the equation of motion for the quasi-charge. We find

$$
L \ddot{\hat{q}}+\partial \epsilon_{0}(q) / \partial q=V_{b}+\delta \hat{V},
$$

where the operator

$$
\delta \hat{V}=-\frac{\hbar}{2 e} \sum_{i=1}^{\infty} \lambda_{i} \dot{\vec{\Xi}}_{i} .
$$

The bath positions satisfy the equation of motion

$$
\ddot{\hat{\Xi}}_{i}+\tilde{\omega}_{i}^{2} \hat{\Xi}_{i}=\lambda_{i} \hbar \dot{\hat{q}} / 2 e .
$$

Direct integration of Eq. (19) yields the sum of the homogeneous and the particular solution, substitution into Eq. (18) yields

$$
\delta \hat{V}=\hat{v}(t)-\int_{0}^{t} Z\left(t-t^{\prime}\right) \dot{\hat{q}}\left(t^{\prime}\right) .
$$

Similar to the situation discussed above for the operator $\delta \hat{I}$, the first term $\hat{v}(t)$ is the voltage noise related to the uncertainty on the level of the initial conditions for the homogeneous solution of Eq. (19). The second term is related to the particular solution of Eq. (19) and describes the response of the bath to an applied current $\dot{\hat{q}}$ through the retarded impedance $Z(t)$. The Fourier transform of the latter is

$$
Z(\omega)=\left(\frac{\hbar}{2 e}\right)^{2} \sum_{i=1}^{\infty} \lambda_{i}^{2} \frac{i \omega}{(\omega+i \eta)^{2}-\tilde{\omega}_{i}^{2}} .
$$

If we choose the bath parameters $\lambda_{i}$ and $\tilde{\omega}_{i}$ such that

$$
\Re \mathrm{e}[Z(\omega)]=\pi\left(\frac{\hbar}{2 e}\right)^{2} \sum_{i=1}^{\infty} \lambda_{i}^{2} \tilde{\omega}_{i} \delta\left(\omega^{2}-\tilde{\omega}_{i}^{2}\right)=R,
$$

the bath's response is ohmic corresponding to that of a resistance $R$. Assuming the bath's initial state to be a canonical equilibrium one, we find that the voltage noise is characterized by the two lowest cumulants, $\langle\hat{v}\rangle=0$ and $\langle\{\hat{v}(t), \hat{v}(0)\}\rangle / 2$, the latter satisfying the fluctuationdissipation theorem,

$$
\begin{aligned}
S_{v}(\omega) & \equiv \int d t e^{i \omega t}\langle\{\hat{v}(t), \hat{v}(0)\}\rangle / 2 \\
& =\hbar \omega \Re \mathrm{e}[Z(\omega)] \operatorname{coth}\left(\hbar \omega / 2 k_{B} T\right) \\
& =\hbar \omega R \operatorname{coth}\left(\hbar \omega / 2 k_{B} T\right) .
\end{aligned}
$$

Now consider the limit $E_{J} \gg E_{C}$, where $\epsilon_{0}(q)=$ $-\Delta_{0} \cos \pi q / e$ corresponds to a purely sinusoidal band in quasi-charge representation with a bandwidth given by 21

$$
\Delta_{0}=16 \sqrt{E_{J} E_{C} / \pi}\left(E_{J} / 2 E_{C}\right)^{1 / 4} e^{-\sqrt{8 E_{J} / E_{C}}} .
$$

Then, together with the choice (22) for the oscillator bath parameters, Eq. 177 takes the form

$$
L \ddot{\hat{q}}+R \dot{\hat{q}}+V_{c} \sin \pi q / e=V_{b}+\hat{v},
$$

where $V_{c}=\pi \Delta_{0} / e$ is the critical voltage. If both sides of Eq. 25) are divided by $V_{c}$, we obtain the dimensionless form

$$
d^{2} \hat{\bar{q}} / d \tau^{\prime 2}+\rho d \hat{\bar{q}} / d \tau^{\prime}+\sin \hat{\bar{q}}=\bar{V}_{b}+\hat{\bar{v}} .
$$

Here, $\tau^{\prime}=\omega_{c} t$ with $\omega_{c}=\left(\pi V_{c} / e L\right)^{1 / 2}$ dual to the plasma frequency, $\hat{\bar{q}}=\pi \hat{q} / e$, the dimensionless resistance $\rho=$ $R\left(e / \pi V_{c} L\right)^{1 / 2}, \bar{V}_{b}=V_{b} / V_{c}$ and $\hat{\bar{v}}=\hat{v} / V_{c}$.

Comparing the quantum Langevin equation Eq. (25) with the corresponding one for the phase dynamics, Eq. (9), we conclude that they are exactly dual to each other. In other words, Eqs. (25) and (9) map onto each other when exchanging the role of quasi-charge $\hat{q}$ and phase $\hat{\phi}$, such that $\pi \hat{q} / e \rightarrow \hat{\phi}$, accompanied by the duality transformations $e / \pi \rightarrow \hbar / 2 e, L \rightarrow C, R \rightarrow 1 / R$, and $V \rightarrow I$. This establishes the exact duality between the two circuits, Fig. 1a and b.

Equation 25 has a simple physical interpretation in the classical limit: it describes a fictitious charge-particle with inertia $L$, provided by the inductor. The sum of the applied bias voltage $V_{b}$ and the resistor-induced noise $v$ drops over the series configuration formed by the junction, the inductor and the resistor. The chargeparticle moves in a tilted washboard potential $U(q)=$ $-\delta_{0} \cos \pi q / e-V_{b} q$ and experiences velocity-proportional damping $R \dot{q}$.

As for its dual counterpart, the classical interpretation of the operator equation (25) hinges on the replacement of the operators $\hat{q}$ and $\hat{v}$ by their respective expectation values $q$ and $v$. This means in particular that we must be allowed to replace $\langle\sin \pi \hat{q} / e\rangle$ by $\sin \langle\pi \hat{q} / e\rangle=\sin \pi q / e$. For this to be correct, the uncertainty in the charge $\delta q$ must be small compared to the elementary charge $e$. The classical version of (25) then describes the motion of a 
narrow wave packet of width $\delta q \ll e$. In phase representation this is consistent with the realization of an extended Bloch state.

As for the case of phase dynamics, it is useful to distinguish two situations, according the value of the parameter $\rho$ in Eq. 26): overdamped charge dynamics, corresponding to $\rho>1$ and underdamped charge dynamics, $\rho<1$.

In the overdamped case, the charge dynamics is always classical as damping times are naturally short, of the order of $L / R$. Note that $L$ here constitutes an additional element in addition to the junction, unlike the case of phase dynamics discussed above where the capacitance $C$ is a property intrinsic to the junction. Overdamped charge dynamics is generally achieved in a high-resistance environment, which favors charge localization. Classical charge dynamics also requires that the environmental noise operator $\hat{v}$ can be treated classically ${ }^{7}$. This implies working in the high-temperature limit $k_{B} T>\hbar R / L$, such that $\hat{v}$ can be replaced by a c-number with $\langle v\rangle=0$ and $\langle v(t) v(0)\rangle=2 k_{B} T R \delta(t)$ in accordance with Eq. 223. The resulting classical Langevin equation can be analyzed through the corresponding Fokker-Planck equation $18 \mid 23$.

In the underdamped case, damping times are long and wave packet spreading becomes important. This issue is particularly important when the charge-particle is in the running state corresponding to a finite current through the junction. In the absence of damping, the uncertainty $\delta q$ can be kept within limits by applying classical timedependent external forces. The spreading of wave packets is governed by a rate proportional to the kinetic energy. For the fictitious charge particle this corresponds to the inductive energy $E_{L}=\Phi_{0}^{2} / 2 L$, which is in fact the energy scale relevant for phase localization related to the Josephson effect. We require the time-dependence of the external force to be fast on the scale $\hbar / E_{L}$. Physically this means that we work under conditions where phase slip events are not suppressed. For example, in the case of microwaves discussed in Section IV below, this implies their frequency to be larger than $E_{L} / \hbar$.

We conclude this section by summarizing the conditions under which the above exact duality is obtained. First of all, we assume the quasi-charge dynamics of the voltage-biased circuit Fig. 1 $1 \mathrm{~b}$ to be determined by the lowest Bloch band only. The lowest band is separated from the next one by the plasma frequency $\hbar \omega_{p}=$ $\sqrt{8 E_{J} E_{C}}$. Since we do not consider here inter-band transitions, we thus assume all energies to be smaller than $\omega_{p}$. Second, in order for the Bloch band to be purely sinusoidal, we need to impose the condition $E_{J} \gg E_{C}$ for the voltage-biased junction of Fig. 1p.

\section{JOSEPHSON JUNCTION CHAIN}

In the previous section we have found that a voltagebiased Josephson junction with a large ratio $E_{J} / E_{C}$ in se-

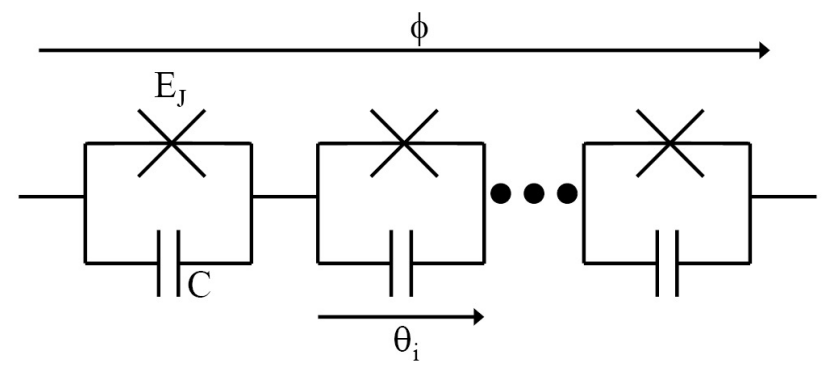

FIG. 4: Josephson junction chain.

ries with an inductance constitutes a phase-slip element. We also saw that the observation of well-defined quasicharge dynamics requires charge-fluctuations very much smaller than a single charge $e$, a condition that can be obtained by a large inductance near the Josephson junction. Experimentally it is not so simple to realize large magnetic inductances very close to the sample. An alternative is to fabricate a large kinetic inductance using a superconductor, and in particular a Josephson junction chain 72426 . It seems therefore quite natural to analyze the possibility to realize a phase-slip junction from a Josephson junction chain, which we investigate in this section. The central idea is that the phase-slip itself occurs on only one of the junctions of the chain; the phases on the other junctions just perform small Josephson oscillations, thereby providing the necessary inductance. In order to demonstrate this idea, we will closely follow the paper by Matveev et al. ${ }^{27}$, who studied the low-energy properties of Josephson junction chains in terms of quantum phase slips.

We start our analysis by considering the Josephson junction chain depicted in Fig. 4. It is a series arrangement of $N$ identical junctions, each with Josephson energy $E_{J}=\hbar I_{c} / 2 e$ and charging energy $E_{C}=e^{2} / 2 C$. Let $Q_{i}$ be the charge on the $i$-th junction and $\theta_{i}$ the conjugate phase difference. We consider the nearest-neighborcapacitance limit and assume the chain to be subjected to an external phase $\phi$. The Hamiltonian can then be written as

$$
H_{\mathrm{ar}}=\sum_{i=1}^{N}\left[4 E_{C}\left(Q_{i} / 2 e\right)^{2}+E_{J}\left(1-\cos \theta_{i}\right)\right] ; \sum_{i=1}^{N} \theta_{i}=\phi .
$$

Ignoring the charging energy for the moment, we find the classical ground state configuration of the chain's phases $\theta_{i}$ by minimizing the Josephson coupling energy, thereby satisfying the constraint. The corresponding configuration can be easily found in the limit $N \gg 1$ and is given by $\theta_{i}=\phi / N$. The resulting Josephson energy hence reads $E_{0}=E_{J} \phi^{2} / 2 N$. This is the inductive energy stored in the chain; the corresponding effective inductance is given by $L_{\mathrm{ar}}=\hbar N / 2 e I_{c}$.

Now consider a phase slip event occurring on one of the junctions, say the $j$ th junction, such that $\theta_{j} \rightarrow \theta_{j}+2 \pi$. 
Due to its periodicity as a function of $\theta_{j}$, the Josephson energy of the junction $j$ does not change. However, the constraint $\sum_{i} \theta_{i}=\phi$ is violated after such a phase-slip event. In order to accommodate the phaseslip without violating the constraint, the phase differences $\theta_{i}$ over the other junctions change slightly, from $\phi / N$ to $(\phi-2 \pi) / N$. Correspondingly, the Josephson energy of the chain changes from $E_{0}=E_{J} \phi^{2} / 2 N$ to $E_{1}=E_{J}(\phi-2 \pi)^{2} / 2 N$. In a similar way one shows that the classical energy needed to accommodate $m$ phaseslips without violating the constraint is given by $E_{m}=$ $E_{J}(\phi-2 \pi m)^{2} / 2 N$. We thus conclude that the ground state of the chain generally is one that contains a fixed number of phase slips for almost any value of the external phase $\phi$, except for the special values $\phi=\pi(2 m+1)$ where the energies $E_{m}$ and $E_{m+1}$ are degenerate. Quantum fluctuations induced by the small but finite charging energy $E_{C}$ lift this degeneracy: they give rise to a nonvanishing amplitude $\Delta_{0}$ for a phase-slip event to occur. We denote the state of the chain with $m$ phase slips by $|m\rangle$. Taking into account the fact that a phase slip can take place on any of the $N$ junctions, we can write the total Hamiltonian for the chain as

$$
\hat{H}_{\mathrm{ar}}=\frac{E_{J}}{2 N}(2 \pi \hat{m}-\phi)^{2}-\frac{N \Delta_{0}}{2} \sum_{m}[|m+1\rangle\langle m|+h . c .] .
$$

Next introduce the operator $\hat{q}$, conjugate to the phaseslip number $\hat{m}$. Specifically, $[\hat{q}, \hat{m}]=-i e / \pi$ such that the operator $e^{i \pi \hat{q} / e}$ is a raising operator with $e^{i \pi \hat{q} / e}|m\rangle=$ $|m+1\rangle$. Using this representation, the Hamiltonian becomes

$$
\hat{H}_{\mathrm{ar}}=\left(E_{J} / 2 N\right)(2 \pi \hat{m}-\phi)^{2}-N \Delta_{0} \cos \pi \hat{q} / e .
$$

A physical interpretation of the operator $\hat{q}$ can be obtained by calculating $\dot{\hat{q}}$; the result reads $\dot{\hat{q}}=$ $(2 e / \hbar) d \hat{H} / d \phi$, which by definition is the operator corresponding to the total current through the chain. Hence, in analogy with the result (15), $\hat{q}$ can be interpreted as the global charge of the chain. It is easy to see that Hamiltonian Eq. 29) has the same form as the quasicharge representation of Hamiltonian $\hat{H}_{0}^{\prime}$ for a single junction in series with an inductor, presented in the previous section. It is therefore straightforward to analyze the case of a voltage-biased chain embedded in a resistive series environment, repeating the steps presented in Section IIB. One finds that the quasi-charge dynamics is governed by the equation

$$
L_{\mathrm{ar}} \ddot{\hat{q}}+R \dot{\hat{q}}+V_{c, \text { ar }} \sin \pi \hat{q} / e=V_{b}+\hat{v},
$$

where $V_{c, \text { ar }}=N V_{c}$ and we used the fact that $\dot{\phi}_{b}=2 e V_{b} / \hbar$. This result can also be presented in the dimensionless form (26), with $\omega_{c, \text { ar }}=\left(\pi V_{c, \text { ar }} / e L_{\mathrm{ar}}\right)^{1 / 2}=\left(2 \pi V_{c} I_{c} / \hbar\right)^{1 / 2}$ and $\rho=R\left(e / \pi V_{c, \mathrm{ar}} L_{\mathrm{ar}}\right)^{1 / 2}=(R / N)\left(2 e^{2} I_{c} / \pi \hbar V_{c}\right)^{1 / 2}$. This result reflects the intuitive argument discussed at the beginning of this section: the inertia $L_{\mathrm{ar}}$ of the charge dynamics is provided by the chain itself. Moreover, it is given by $N$ times the nonlinear inductance $\hbar / 2 e I_{c}$ of a single junction in the chain. This means that $L_{\mathrm{ar}}$ can be tuned in principle, either by tuning $N$ or by using SQUID loops instead of single junctions as in ${ }^{7}$, such that $I_{c}$ can be tuned with a magnetic flux. We also note that the critical voltage of a Josephson junction chain is $N$ times larger than the one of a single Josephson junction. This is relevant for the discussion in the next section, where we will analyze the $I-V$ characteristic of a phase-slip junction under microwave irradiation. As the width of the appearing current steps scales with the critical voltage of the phase-slip junction, a Josephson junction chain has necessarily larger current-steps that are as a consequence more robust against voltage noise.

\section{PHASE-SLIP JUNCTION UNDER THE INFLUENCE OF MICROWAVE IRRADIATION}

As it was already mentioned in the Introduction, it is of interest to study the behavior of phase-slip junctions under the influence of microwaves. In view of duality, we expect the current-voltage characteristics to exhibit steps at well-defined values of the current that are multiples of the microwave frequency, so-called current Shapiro steps. In order to demonstrate this, we have numerically integrated Eq. 25) in the classical limit. We set $V_{b}(t)=V+V_{\mathrm{MW}} \sin \left(\omega_{\mathrm{MW}} t\right)$, and ignore the effect of fluctuations. The results are plotted in Figs. 5 and 6 for two choices of the dimensionless damping parameter $\rho$, corresponding to the overdamped and underdamped limit, respectively.

Figures $5 \mathrm{a}$ and $6 \mathrm{a}$ show the current-voltage characteristics in the absence of microwaves, $V_{\mathrm{MW}}=0$. In the overdamped case, Fig. 5 a, the current remains zero as long as the voltage $V$ is smaller than the critical voltage $V_{c}$ : the phase-slip junction is in the Coulomb blockade regime. Beyond $V_{c}$, the current rapidly increases until it reaches the linear asymptote $I \sim V / R$, where the junction is in the superconducting state and the voltage drops entirely over the resistor. This characteristic corresponds in fact to the so-called Bloch nose, which has been studied in the experiment $t^{7}$ in the presence of thermal fluctuations. It was found that such fluctuations induce a smooth interpolation between the Coulomb blockade and the superconducting branch, in agreement with theory ${ }^{23}$. In the underdamped situation, Fig. 6a, the two branches in the characteristic co-exist in the Coulomb blockade region. Since both branches are accessible, we generally expect to find hysteretic behavior of the phase-slip junction. To the best of our knowledge, this limit has not yet been accessed experimentally. The behavior shown in Figs. 5 a and 6 a have a dual counterpart in the usual RCSJ-model, where both the overdamped and the underdamped limit have been studied in experiments 8115 .

If microwaves are applied, steps appear at well-defined values of the current that are multiples of the applied 

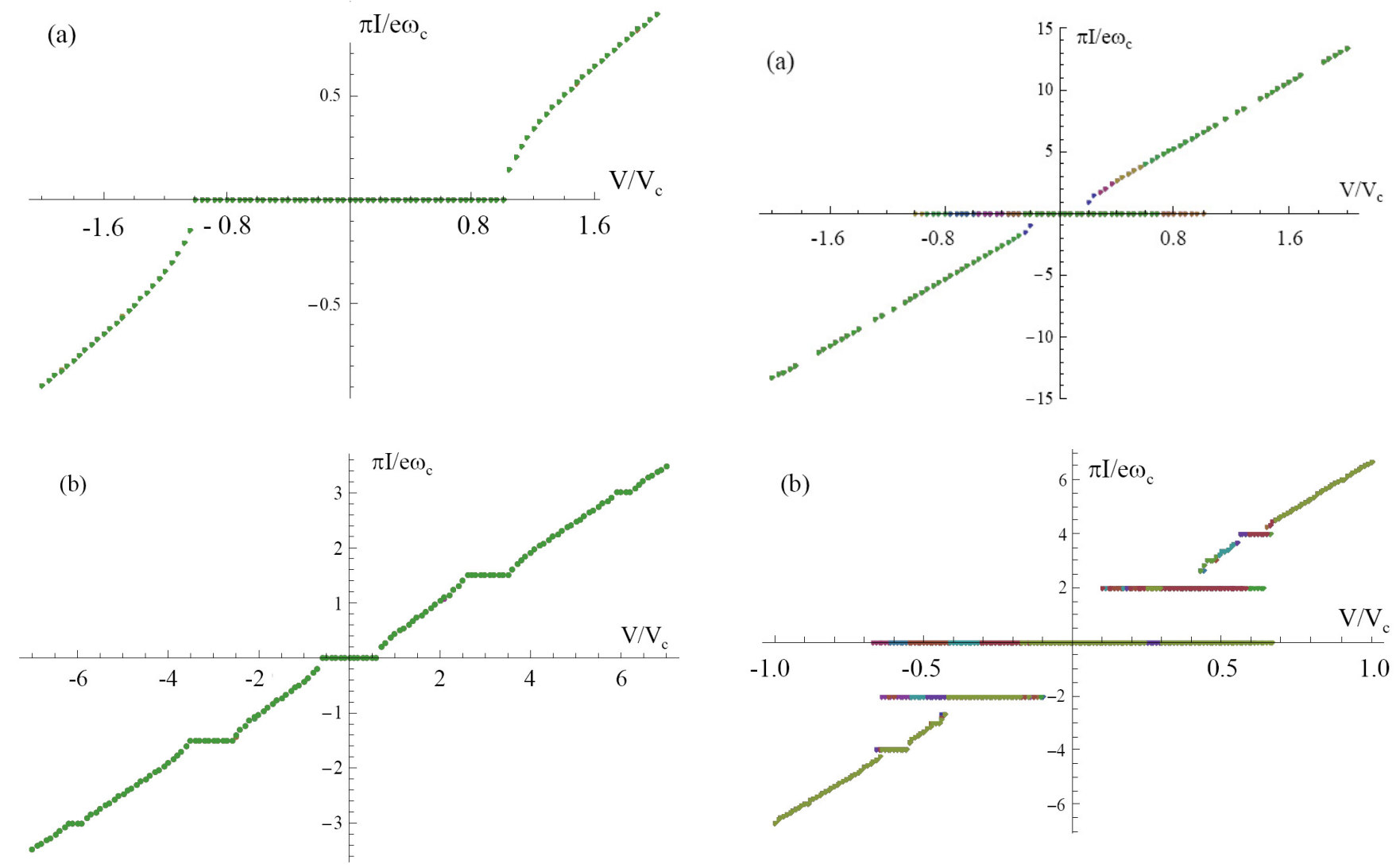

FIG. 5: Current-voltage characteristics for an overdamped phase-slip junction with $\rho=2$, (a) without MW irradiation, (b) under MW-irradiation with amplitude $V_{\mathrm{MW}}=5 V_{c}$ and frequency $\omega_{\mathrm{MW}}=1.5 \omega_{c}$.

MW frequency, as can be seen in Figs. 5b and 6b. The steps are clearly visible; they are integrated within the overall $I-V$ characteristic, the average slope of which remains determined by the resistance $R$. In the overdamped case, there is a one-to-one correspondence between current and voltage, as for the case without MW. In fact, the shape of each step in Fig. 5b appears to be a replica of the characteristic in the absence of $\mathrm{MW}$, Fig. 5a. The one-to-one correspondence between voltage and current found in the overdamped limit is lost in the underdamped case: various current steps appear within the same voltage interval. In order to obtain the result shown in Fig. 6b, Eq. 25) had to be integrated for a range of initial conditions on the quasi-charge for each value of the DC voltage $V$. Note that the resistive branch is absent in the regions of overlapping steps; this is of interest as it possibly makes it easier to lock on a given step in the experiment.
FIG. 6: Current-voltage characteristics for an underdamperd phase-slip junction with $\rho=0.15$, (a) without MW irradiation, (b) under MW-irradiation with amplitude $V_{\mathrm{MW}}=3 V_{c}$ and frequency $\omega_{\mathrm{MW}}=2 \omega_{c}$.

\section{CONCLUSIONS AND OUTLOOK}

We have studied two circuits, a single Josephson junction in an inductive-resistive environment and a Josephson junction chain, in view of the realization of the dual of the Josephson effect. In both cases we elucidate the importance of the role of the inductance in order to reduce charge fluctuations. Duality between these two circuits and a single Josephson junction is valid in the quantum and classical regime. Here, we derived $I(V)$ characteristics in the classical quasi-charge regime. In case of larger quasicharge fluctuations going beyong the classical regime, perturbation theory can be applied in analogy to the $P(E)$ theory in Josephson junctions governed by phase-dynamic $\$ 1728129$.

Until now there are only a few experiments dealing with quasi-charge dynamics724/25/30. We believe that both circuits are experimentally feasible and they are of particular interest in terms of the realization of current Shapiro steps. The successful realization of such an experiment would link the frequency to the current by a quantum electrical recipe and close the metrological triangle. Ultimately, the quantum metrological triangle experiment would enable a consistency check of the 
fundamental constants of nature, the electron charge $e$ and Planck's constant $h$, and as a consequence link the kilogram (the only unit still defined on the basis of one artefact, prototype of the mass kept in metrology institutions), to the Planck constant. The circuit based on a single Josephson chain seems from our point of view the most promising as it enables to realize large current steps.

\section{Acknowledgments}

We wish to thank O. Buisson, N. Didier, L. Glazman, D. Haviland, L. Kuzmin, and A. Zaikin for valuable dis- cussions. Financial support from the European Community (STREP MIDAS) and the French National Research Agency (ANR QUANTJO) is gratefully acknowledged. FH thanks the ICTP Trieste (Italy), where part of the work described here has been performed, for hospitality.
1 R. Savit, Rev. Mod. Phys. 52, 453 (1980).

2 A. Schmid, Phys. Rev. Lett. 51, 1506 (1983).

3 G. Schön and A.D. Zaikin, Phys. Rep. 198, 237 (1990)

4 U. Weiss, Quantum Dissipative Systems, World Scientific, Singapore, 1999.

${ }^{5}$ G.-L. Ingold and H. Grabert, Phys. Rev. Lett. 83, 3721 (1999).

6 S.M. Apenko, Phys. Lett. A 142, 277 (1989).

7 S. Corlevi, W. Guichard, F.W.J. Hekking, and D.B. Haviland, Phys. Rev. Lett. 97, 096802 (2006).

8 A. Steinbach, P. Joyez, A. Cottet, D. Esteve, M. H. Devoret, M. E. Huber, and John M. Martinis, Phys. Rev. Lett. 87, 137003 (2001).

${ }^{9}$ S. Shapiro, Phys. Rev. Lett. 11, 80 (1963).

10 R.L. Kautz, Rep. Prog. Phys. 59, 935 (1996).

11 Clark A. Hamilton, Review of Scientific Instruments 71, 3611 (2000); J. Niemeyer in Handbook of Applied Superconductivity, edited by B. Seeber (Institute of Physics, Philadelphia, PA, 1998), Vol 2, P. 1813.

12 J.E. Mooij and Yu. V. Nazarov, Nature Physics 2, 169 (2006).

13 A.D. Zaikin, D.S. Golubev, A. van Otterlo and G.T. Zimányi, Phys. Rev. Lett. 78, 1552 (1997)

14 K.Y. Arutyunov KY, D.S. Golubev and A.D. Zaikin, Phys. Rep. 464, 1 (2008).

15 M. Tinkham, Introduction to Superconductivity, McGrawHill, New-York, 1996.

16 A.O. Caldeira and A.J. Leggett, Phys. Rev. Lett. 46, 211 (1981); Ann. Phys. (NY) 149, 371 (1983).

17 G.-L. Ingold and Yu.V. Nazarov, in Single Charge Tunnelling, edited by H. Grabert and M. H. Devoret, NATO ASI Series B, Vol. 294, pp. 21-107 (Plenum Press, New
York, 1992).

18 N.G. van Kampen, Phys. Rep. 24, 171 (1976).

19 Y.M. Ivanchenko and L.A. Zilberman, Sov. Phys. JETP 28, 1272 (1969).

${ }^{20}$ D.V. Averin, A.B. Zorin and K.K. Likharev, Sov. Phys. JETP 61, 407 (1985).

${ }^{21}$ K.K. Likharev and A.B. Zorin, J. Low Temp. Phys. 59, 347 (1985).

22 E.M. Lifshitz and L.P. Pitaevskii Statistical Physics, Part 2, Landau and Lifshitz Course of Theoretical Physics Volume 9, Pergamon Press Oxford, 1980.

23 The Fokker-Planck approach has been applied to the case of overdamped quasi-charge dynamics, see I.S. Beloborodov, F.W.J. Hekking, and F. Pistolesi, in New Directions in Mesoscopic Physics (Towards Nanoscience), edited by R. Fazio, V.F. Gantmakher and Y. Imry, p. 339 (Kluwer Academic Publishers, Dordrecht, 2003).

24 M. Watanabe and D.B. Haviland, Phys. Rev. Lett.86, 5120 (2001).

25 F. Nguyen, N. Boulant, G. Ithier, P. Bertet, H. Pothier, D. Vion, D. Esteve, Phys. Rev. Lett. 99, 187005 (2007).

26 V.E. Manucharyan, J. Koch, L. Glazman, and M. Devoret, arXiv:0906.0831

27 K.A. Matveev, A.I. Larkin, and L.I. Glazman, Phys. Rev. Lett. 89, 096802 (2002).

28 D.V. Averin, Yu.V. Nazarov and A.A. Odintsov, Physica B 165 \& 166, 945 (1990).

29 A. Zazunov, N. Didier, and F.W.J. Hekking, Europhys. Lett. 83, 47012 (2008).

30 L.S. Kuzmin and D.B. Haviland, Phys. Rev. Lett. 67, 2890 (1991). 